\title{
Medical Image of the Week: Cytomegalovirus Pneumonia
}

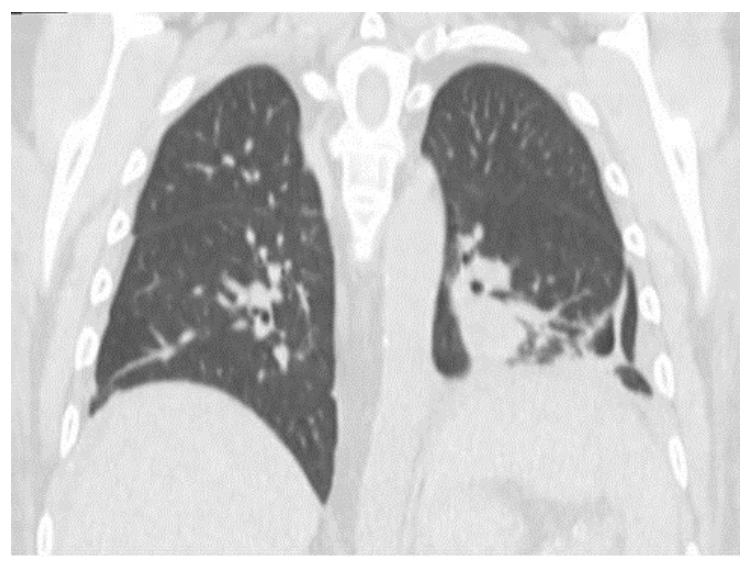

Figure 1. CT Chest, coronal cut showing left lower lobe consolidation.

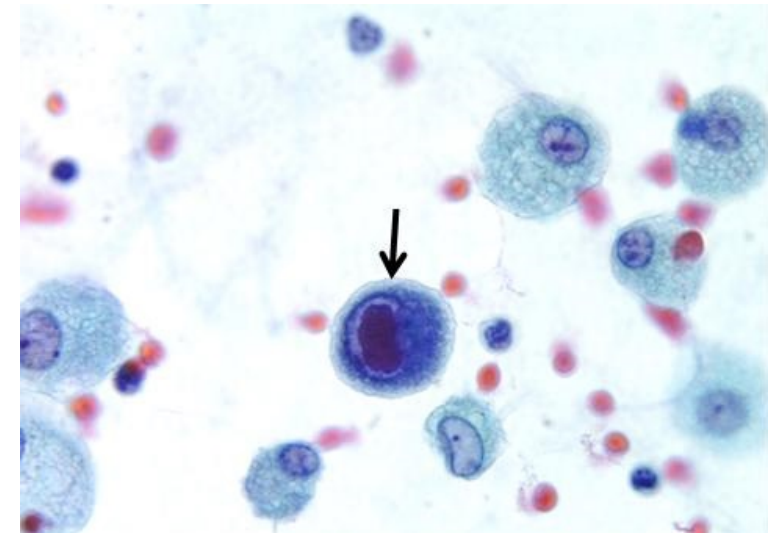

Figure 2. Pap stain highlights an enlarged cytomegalovirus-infected pneumocyte containing a single, dark intranuclear inclusion (arrow) with surrounding halo, giving the cell a characteristic "owl's eye" appearance. Background cells consist of predominantly of macrophages and red blood cells (100x).

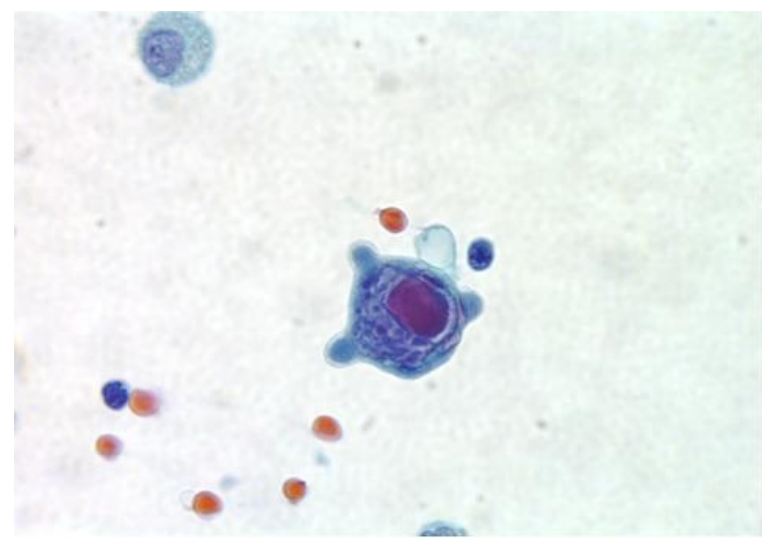


Figure 3. CMV-infected pneumocyte demonstrating an enlarged nucleus with a single dense intranuclear inclusion. The cytoplasm contains smaller basophilic inclusions with vacuolization and cytoplasmic projections (Pap stain, 100x).

A 29 year-old female with a history of systemic lupus erythematosus presented with a seven-day history of fever, dyspnea and a non-productive cough. She underwent renal transplantation four weeks prior to admission and was maintained on mycophenolate, tacrolimus, prednisone and prophylactic fluconazole, trimethoprim/sulfamethoxazole and valgangcyclovir. A CT chest was performed (Figure 1) and revealed left lower lobe consolidation. A BAL was performed in the left lower lobe and the cell count revealed $50 \%$ lymphocytes, $13 \%$ neutrophils and $37 \%$ macrophages. The BAL Papanicolaou stain showed enlarged cytomegalovirus-infected pneumocytes with the characteristic "owl's eye" appearance (Figures 2 and 3). CMV quantitative PCR from serum resulted $648,615 \mathrm{IU} / \mathrm{m}$. The BAL culture grew CMV. The patient was started on treatment with valgangcyclovir with clinical improvement.

While often thought of as a "pneumonitis" with diffuse infiltrates, CMV can cause a lobar pneumonia in up to $30 \%$ of patients. Prophylaxis is effective, but cases can occur despite a preemptive strategy.

Nathaniel Reyes MD*, Julianna J. Weiel MSII+, Erika R. Bracamonte MD ${ }^{+}$, Linda Snyder $\mathrm{MD}^{*}$

Department of Medicine, Division of Pulmonary and Critical Care Medicine*

Department of Pathology ${ }^{+}$

University of Arizona

Tucson, Arizona

\section{Reference}

Kang E, Patz E, Miller NL. Cytomegalovirus pneumonia in transplant patients: CT findings. J Comput Assisted Tomogr. 1998:20:295-9. [CrossRef] 